\title{
Analysis of T Cells Using Flow Cytometry
}

\author{
Insoo Kang
}

Department of Internal Medicine, Yale University School of Medicine, New Haven, Connecticut 06520, USA

$T$ cells are an essential cellular component of the immune system. When $T$ cells encounter antigen and receive co-stimulatory signals, they become activated, proliferate and produce cytokines. Flow cytometry is a valuable research tool in analyzing $T$ cell functions including pro-

\section{Introduction}

$\mathrm{T}$ cells are an essential cellular component of adaptive immunity. The progenitors for $\mathrm{T}$ cells are developed in the bone marrow and migrate to the thymus where they become immature $\mathrm{T}$ cells or thymocytes $(1,2)$. In the thymus, immature $\mathrm{T}$ cells undergo positive and negative selection processes. A small number of immature $\mathrm{T}$ cells which have survived these processes are exported into the circulation as mature but naïve $\mathrm{T}$ cells $(2,3)$. When naïve $\mathrm{T}$ cells recognize antigen in the context of the major histocompatibility complex (MHC) and receive co-stimulation from antigen-presenting cells (APCs) in the secondary lymphoid tissues (e.g. lymph nodes), naïve $\mathrm{T}$ cells become activated, proliferate and differentiate into effector cells (2). When the source of antigen (e.g. microbial infection) is cleared, most effector $\mathrm{T}$ cells undergo activation-induced cell death while a few cells survive and become memory $\mathrm{T}$ cells, providing immune memory.

$\mathrm{T}$ cells can be divided into CD4+ and CD8+ $\mathrm{T}$ cells based on the expression of CD4 and CD8 which bind MHC class II and I molecules, respectively. Using T cell receptors (TCR), CD4+ $\mathrm{T}$ cells recognize antigen presented in the context of MHC class II molecules while CD8+ $\mathrm{T}$ cells recognize antigen presented in the restriction of MHC class I molecules. CD4+ and CD8+ $\mathrm{T}$ cells have distinct roles although both cell liferation, survival and cytotoxicity as well as cytokine production and cell signaling. This article has reviewed the utility of flow cytometry in evaluating $T$ cell functions. Key Words. T cells, Flow cytometry

populations can produce cytokines. CD4 $+\mathrm{T}$ cells are called $\mathrm{T}$ helper (Th) cells in that the primary function of CD4+ $\mathrm{T}$ cells is to help other immune cells like B cells and innate immune cells by producing cytokines and expressing co-stimulatory molecules $(1,4)$. Th cells can be further divided into Th1, Th2 and Th17 cells that dominantly produce IFN- $\gamma$, IL-4 and IL-17, respectively $(2,5)$. CD8+ T cells with the expression of cytotoxic molecules such as perforin can kill host cells infected with microorganisms or tumor cells (6). In fact, $\mathrm{CD} 8+\mathrm{T}$ cells are called cytotoxic $\mathrm{T}$ cells.

Flow cytometry is a research method applied to study the phenotype and function of cells using lasers (reviewed (7)). The technology involved in flow cytometry has significantly evolved over the past decade. Now flow cytometry is considered an essential tool in analyzing $\mathrm{T}$ cells. This paper will review flow cytometric methods used to analyze $\mathrm{T}$ cell functions including cytokine production, cell proliferation, and survival.

\section{Analyzing cytokine production by $\mathrm{T}$ cells}

The frequency and characteristics of CD4+ and CD8+ T cells that produce cytokines in peripheral blood can be measured in vitro using flow cytometry. Whole blood, peripheral blood mononuclear cells (PBMCs), or purified T cells can be stimulated with the antigen of interest or mitogens like phorbol

<Received: April 13, 2013, Accepted: April 20, 2013>

Corresponding to : Insoo Kang, Department of Internal Medicine, Yale University School of Medicine, 300 Cedar Street, New Haven, CT 06520, USA. E-mail : Insoo.kang@yale.edu 
myristate acetate (PMA) and ionomycin (8-12). Commonly used antigens include the ones derived from pathogens such as cytomegalovirus (CMV), Epstein Barr virus (EBV) and influenza virus as well as toxins (e.g. tetanus toxoid) $(8,9,12,13)$. Both antigenic lysate and overlapping peptides of specific antigens can be used. The duration of stimulation can be as short as 4 hours. A chemical blocking the release of proteins from the Golgi complex (e.g. Brefeldin A) is added to enhance intracytoplasmic accumulation of cytokines during cell stimulation. In measuring antigen-specific cytokine production, cells can be treated with ligands for co-stimulatory molecules such as CD28 in addition to antigens $(8,9,14)$. Stimulated cells are fixed and permeabilized for intracellular cytokine staining. Although fixed cells can be also stained with antibodies to cell surface molecules, the epitopes of some cell surface molecules are altered with fixation, resulting in suboptimal staining with antibodies. Thus, in performing experiments, researchers should test individual molecules to determine whether fixation affects cell surface molecule staining with antibodies. Different types of fixation and permeabilization agents are available. Paraformaldehyde $(1 \sim 4 \%)$ and saponin $(0.5 \% \mathrm{v} / \mathrm{v}$ in PBS) based buffers are commonly used as fixation and permeabilizing reagents, respectively $(15,16)$. Also, different brands of reagents for cell fixation and permeabilization are available. In conjunction with cytokine analysis, cellular characteristics of cytokine producing and non-producing $\mathrm{T}$ cells can be simultaneously analyzed using antibodies to cell surface and intracellular molecules. For instance, the early activation marker CD69 can be identified by staining cells with antibodies to CD69. Also, transcription factors that regulate the expression of cytokines (e.g. T-bet) and cytotoxic molecules (e.g. perforin) can be co-stained. Overall, flow cytometric analysis of activated $\mathrm{T}$ cells can be a powerful tool in analyzing $\mathrm{T}$ cells producing cytokines in health and disease. Indeed, our group found an alteration in the frequency of IL-17-producing Th17 cells in patients with systemic lupus erythematosus and older adults using flow cytometry $(10,17)$.

\section{Identification of antigen-specific $\mathrm{T}$ cells using flow cy- tometry}

$\mathrm{T}$ cells have diversity in $\mathrm{T}$ cell receptor (TCR) repertoire that allows recognition of different antigenic peptides. APCs present the antigenic peptides loaded in the MHC class molecules to TCR. Based on this principle, $\mathrm{T}$ cells with the capacity to recognize specific antigenic peptides can be identified by flow cytometry. An MHC class I or II molecule-restricted antigenic peptide can be loaded into the MHC class I or II multimers, typically tetramers (18-21). The peptide-loaded MHC mole- cules can be added to blood cells along with antibodies to other molecules such as CD4 and CD8. TCR specific for the loaded peptide will bind to the complex of the peptide and MHC molecules, conjugated with a fluorescent dye. Subsequently, T cells with TCR specific for the loaded peptide can be identified by flow cytometry. The limitation of this assay is that only specific peptide sequences can be loaded into particular types of MHC class I or II multimers. Thus, this assay cannot be used in analyzing $\mathrm{T}$ cells from individuals who do not have the particular type of MHC molecules. For instance, HLA-A2 tetramers loaded with a particular type of CMV-specific peptides can be used only for studying CD8+ $\mathrm{T}$ cells from individuals with HLA-A2.

Antigen-specific $\mathrm{T}$ cells can be also identified using intracellular cytokine staining. As discussed above, PBMCs or whole blood cells can be stimulated with antigenic protein or peptides $(8,9,12,14)$. Stimulated cells are fixed, permeabilized and stained with antibodies to $\mathrm{T}$ cell markers and cytokines. Flow cytometric analysis can identify CD4+ and CD8+ T cells secreting cytokines such as IFN- $\gamma$. Although this assay can be used independently of MHC molecule types, it is likely that only a portion of antigen-specific $\mathrm{T}$ cells would secrete cytokines. Nevertheless, this assay can be a useful tool in assessing the frequency of CD4+ and CD8+ $\mathrm{T}$ cells specific for different antigens.

\section{Measuring T cell proliferation, survival and cytotoxicity using flow cytometry}

$\mathrm{T}$ cell proliferation was traditionally measured using tridium thymidine with a risk of radiation exposure. Now $\mathrm{T}$ cell proliferation can be assessed using fluorescent dyes, most commonly carboxyfluorescein succinimidyl ester (CFSE) which incorporates into the cytoplasm by covalent binding to intracellular molecules $(22,23)$. T cells are stained with CFSE and then stimulated with mitogens like anti-CD3/CD28 antibodies or antigenic peptide in the presence of APCs. After cell culture, cells are stained with antibodies to $\mathrm{T}$ cells. Proliferating $\mathrm{T}$ cells can be then identified by flow cytometry (23). With each cell division, the intensity of CFSE reduces by half. The results of this assay include the percent of proliferating cells and the number of cell divisions that have occurred in stimulated samples.

$\mathrm{T}$ cell survival can be also assessed using flow cytometry (24-26). Cultured T cells or PBMCs can be stained with appropriate cell surface markers as well as with annexin $\mathrm{V}$ and 7-aminoactinomycin D (7-AAD) $(24,25)$. Annexin V is a cellular protein that binds phospholipids like phosphatidylserine (PS) expressed on the outer surface of the cell membrane 
upon cell death in a calcium dependent manner (26). In live cells, PS is found within the cell membrane but not on the outer surface. During early apoptosis (activation-induced cell death) PS is exposed on the outer surface of the cell membrane, which allows annexin $\mathrm{V}$ to bind PS (26). 7-AAD is a fluorescent chemical with the strong capacity to bind DNA (25). 7-AAD can penetrate dead or dying cells with the compromised cell membrane and bind DNA while such penetration and binding can not occur in live cells with the intact cell membrane. Annexin V conjugated with a fluorescent dye and 7-AAD are added to cultured T cells or PBMCs. Cells are then analyzed on a flow cytometer. Live (annexin $\mathrm{V}$ - and 7-AAD-) and dead cells (annexin $\mathrm{V}+$ and 7-AAD+/-) can be identified. In place of 7-AAD, propidium iodide (PI), which also binds DNA, can be used.

CD8+ $\mathrm{T}$ cells kill virus-infected cells or tumor cells using cytotoxic molecules. The radioisotope Chromium-51 $\left({ }^{51} \mathrm{Cr}\right)$ has been used in measuring the cytotoxicity of $\mathrm{CD} 8+\mathrm{T}$ cells. However, recent studies have utilized CFSE in analyzing the cytotoxicity of CD8 $+\mathrm{T}$ cells $(27,28)$. Target cells are stained with CFSE and co-cultured with effector cells (27). 7-AAD or PI is added to cell culture. Stained cells are then analyzed on a flow cytometer to identify dead target cells based on CFSE and 7-AAD or PI staining. In analyzing human immunodeficiency virus (HIV)-specific CD8 $+\mathrm{T}$ cell cytotoxicity, the sensitivity and specificity of the CFSE-based assay were compatible to those of the traditional Cr51 release assay (27).

\section{Analyzing cell signaling molecules by flow cytometry}

Cell signaling molecules, such as the signal transducer and activator of transcription (STAT) and nuclear factor kappa-light-chain-enhancer of activated B cells (NF- $\kappa$ B), are traditionally analyzed by Western blot. However, recent studies have utilized flow cytometry in analyzing the cell signaling molecules (reviewed in $(29,30)$ ). For instance, the activation (phosphorylation) of STAT5 can be analyzed in T cells treated with the cytokine IL-7 which is known to activate STAT5 (24). The advantage of this assay is to quantify the activation levels of signaling molecules while the results of the Western blot are shown semi-quantitatively (30). Also, cell signaling molecules can be measured in a certain cell subset in PBMCs or whole blood without the cell purification $(30,31)$. An increasing number of antibodies to phosphorylated forms of signaling molecules are available for flow cytometric cell signaling analysis. Whole blood cells, PBMCs or purified $\mathrm{T}$ cells are stimulated. Stimulated cells are fixed, permeablized and stained with antibodies to phosphorylated (activated) cell signaling molecules. Different permeabilization reagents have ad- vantages and disadvantages. In general, alcohol-based permebailization reagents (e.g. $90 \%$ ice-cold methanol) provide the most optimal result for intracellular phosphor protein analysis. However, this treatment can compromise cell surface staining by affecting antigenic epitopes recognized by antibodies (30). This point should be carefully considered when designing flow cytometric analysis of cell signaling molecules. Cells stained with appropriate antibodies are analyzed on a flow cytometer. The mean fluorescent intensity of a cell signaling molecule or the percent of cells positive for the cell signaling molecule can be compared among cells treated in different conditions (e.g. cytokine vs. vehicle control) (30).

\section{Conclusion}

With the advancement of technology, the functional analysis of human $\mathrm{T}$ cells with a limited blood volume becomes more feasible. In particular, functions of a small subset of $\mathrm{T}$ cells can be analyzed in total PBMCs or whole blood cells using flow cytometry without cell purification. Such analyses include the ones for cell proliferation, survival and cytokine production as well as for cell signaling molecules. These techniques are valuable tools in studying biologic and pathologic roles of $\mathrm{T}$ cells in health and disease.

\section{References}

1. Reiner SL. Development in motion: helper $\mathrm{T}$ cells at work. Cell 2007;129:33-6.

2. Lee N, Shin MS, Kang I. T-cell biology in aging, with a focus on lung disease. J Gerontol A Biol Sci Med Sci 2012;67:254-63.

3. Starr TK, Jameson SC, Hogquist KA. Positive and negative selection of T cells. Annu Rev Immunol 2003;21:139-76.

4. Murphy KM, Stockinger B. Effector $\mathrm{T}$ cell plasticity: flexibility in the face of changing circumstances. Nat Immunol 2010;11:674-80.

5. Shin MS, Lee N, Kang I. Effector T-cell subsets in systemic lupus erythematosus: update focusing on Th17 cells. Curr Opin Rheumatol 2011;23:444-8.

6. Cui W, Kaech SM. Generation of effector CD8+ T cells and their conversion to memory $\mathrm{T}$ cells. Immunol Rev 2010;236:151-66.

7. Soloski MJ, Chrest FJ. Multi-parameter flow cytometry for discovery of disease mechanisms in rheumatic diseases. Arthritis Rheum 2013. [Epub ahead of print].

8. Kang I, Hong MS, Nolasco H, Park SH, Dan JM, Choi $\mathrm{JY}$, et al. Age-associated change in the frequency of memory CD4 $+\mathrm{T}$ cells impairs long term CD4+ $\mathrm{T}$ cell responses to influenza vaccine. J Immunol 2004;173: 673-81.

9. Kang I, Quan T, Nolasco H, Park SH, Hong MS, Crouch $\mathrm{J}$, et al. Defective control of latent Epstein-Barr virus infection in systemic lupus erythematosus. J Immunol 
2004;172:1287-94.

10. Lee JS, Lee WW, Kim SH, Kang Y, Lee N, Shin MS, et al. Age-associated alteration in naive and memory Th17 cell response in humans. Clin Immunol 2011;140:84-91.

11. Lee WW, Kang SW, Choi J, Lee SH, Shah K, Eynon EE, et al. Regulating human Th17 cells via differential expression of IL-1 receptor. Blood 2010;115:530-40.

12. Shin MS, Lee JS, Lee N, Lee WW, Kim SH, Kang I. Maintenance of CMV-specific CD8+ T cell responses and the relationship of IL-27 to IFN- $\gamma$ levels with aging. Cytokine 2013;61:485-90.

13. Kallas EG, Gibbons DC, Soucier H, Fitzgerald T, Treanor JJ, Evans TG. Detection of intracellular antigen-specific cytokines in human $\mathrm{T}$ cell populations. $\mathrm{J}$ Infect Dis 1999;179:1124-31.

14. Suni MA, Picker LJ, Maino VC. Detection of antigen-specific $\mathrm{T}$ cell cytokine expression in whole blood by flow cytometry. J Immunol Methods 1998;212:89-98.

15. Sander B, Andersson J, Andersson U. Assessment of cytokines by immunofluorescence and the paraformaldehyde-saponin procedure. Immunol Rev 1991;119:65-93.

16. Vikingsson A, Pederson K, Muller D. Enumeration of IFN-gamma producing lymphocytes by flow cytometry and correlation with quantitative measurement of IFN-gamma. J Immunol Methods 1994;173:219-28.

17. Shah K, Lee WW, Lee SH, Kim SH, Kang SW, Craft $\mathrm{J}$, et al. Dysregulated balance of Th17 and Th1 cells in systemic lupus erythematosus. Arthritis Res Ther 2010;12:R53.

18. Wölfl M, Schalk S, Hellmich M, Huster KM, Busch DH, Berthold F. Quantitation of MHC tetramer-positive cells from whole blood: evaluation of a single-platform, six-parameter flow cytometric method. Cytometry A 2004;57: 120-30.

19. Nepom GT. MHC class II tetramers. J Immunol 2012; 188:2477-82.

20. Appay V, Rowland-Jones SL. The assessment of antigen-specific CD8+ $\mathrm{T}$ cells through the combination of MHC class I tetramer and intracellular staining. J Immunol Methods 2002;268:9-19.
21. Wooldridge L, Lissina A, Cole DK, van den Berg HA, Price DA, Sewell AK. Tricks with tetramers: how to get the most from multimeric peptide-MHC. Immunology 2009;126:147-64.

22. Parish CR. Fluorescent dyes for lymphocyte migration and proliferation studies. Immunol Cell Biol 1999;77: 499-508.

23. Parish CR, Glidden MH, Quah BJ, Warren HS. Use of the intracellular fluorescent dye CFSE to monitor lymphocyte migration and proliferation. Curr Protoc Immunol 2009; Chapter 4:Unit4.9.

24. Kim HR, Hong MS, Dan JM, Kang I. Altered IL-7Ralpha expression with aging and the potential implications of IL-7 therapy on CD8+ T-cell immune responses. Blood 2006;107:2855-62.

25. Coder DM. Assessment of cell viability. Curr Protoc Cytom 2001; Chapter 9:Unit 9.2.

26. Vermes I, Haanen C, Steffens-Nakken H, Reutelingsperger C. A novel assay for apoptosis. Flow cytometric detection of phosphatidylserine expression on early apoptotic cells using fluorescein labelled Annexin V. J Immunol Methods 1995;184:39-51.

27. Godoy-Ramirez K, Mäkitalo B, Thorstensson R, Sandström E, Biberfeld G, Gaines H. A novel assay for assessment of HIV-specific cytotoxicity by multiparameter flow cytometry. Cytometry A 2005;68:71-80.

28. Zhang Y, Wu XH, Luo CL, Zhang JM, He BC, Chen G. Interleukin-12-anchored exosomes increase cytotoxicity of $\mathrm{T}$ lymphocytes by reversing the JAK/STAT pathway impaired by tumor-derived exosomes. Int $\mathrm{J}$ Mol Med 2010;25:695-700.

29. Schulz KR, Danna EA, Krutzik PO, Nolan GP. Singlecell phospho-protein analysis by flow cytometry. Curr Protoc Immunol 2012; Chapter 8:Unit 8.17.1-20.

30. Suni MA, Maino VC. Flow cytometric analysis of cell signaling proteins. Methods Mol Biol 2011;717:155-69.

31. Chow S, Hedley D, Shankey TV. Whole blood processing for measurement of signaling proteins by flow cytometry. Curr Protoc Cytom 2008; Chapter 9:Unit 9.27. 